no mapping hemipelvis is recommended. Nevertheless, most hemipelvis lymphadenectomies showed no nodal involvement. Previously, we published a preoperative predictive score of nodal involvement. In case of a negative score (value 3-4), the risk of nodal metastases was extremely low. The present multicentre study aims to validate the predictive score of nodal involvement in patients undergoing nodal assessment.

Methods EC patients undergoing surgical treatment with nodal staging were included in the analysis. A preoperative predictive score of nodal involvement was calculated for all patients before surgery was performed. The score included myometrial infiltration, tumor grading $(\mathrm{G})$, tumor diameter, and Ca125 assessment. STARD (standards for Reporting Diagnostic accuracy studies) guidelines were followed for the score accuracy. Results 1038 patients were included in the analysis and 155 (14.9\%) nodal metastases were detected. The score was negative ( 3 and 4) in 475 patients and positive (5-7) in 563 cases. The score showed $83.2 \%$ sensitivity, $50.8 \%$ specificity, $94.5 \%$ negative predictive value, and $55.7 \%$ diagnostic accuracy. The area under the curve (AUC) was 0.75. The logistic regression between negative score and absent nodal metastases showed OR 5.133, 95\% CI (3.30-7.98), p <0.001.

Conclusions The nodal preoperative predictive score is a fair diagnostic test. The risk of nodal metastasis is extremely low in case of negative score. In SLN failure, the application of the present score associated with SLN algorithm could avoid unnecessary lymphadenectomies.

\section{EPV146/\#133 ACCEPTABILITY OF BARIATRIC SURGERY IN YOUNG WOMEN WITH ENDOMETRIAL CANCER AND ATYPICAL ENDOMETRIAL HYPERPLASIA: A QUALITATIVE STUDY}

${ }^{1}$ RS Kim*, ${ }^{1} \mathrm{G}$ Ene, ${ }^{2} \mathrm{~A}$ Simpson, ${ }^{3} \mathrm{D}$ Gesink, ${ }^{1} \mathrm{~S}$ Ferguson. ${ }^{1}$ Princess Margaret Cancer Centre/ University of Health Network/Sinai Health Systems, Gynecologic Oncology, Toronto, Canada; ${ }^{2}$ St.Michael's Hospital/Unity Health Toronto, Obstetrics and Gynecology, Toronto, Canada; ${ }^{3}$ University of Toronto, Dalla Lana School of Public Health, Toronto, Canada

\subsection{6/ijgc-2021-IGCS.216}

Objectives Endometrial cancer (EC) or atypical hyperplasia $(\mathrm{AH})$ in young women with obesity is often the first significant obesity-related comorbidity they experience. Significant, sustained weight loss through bariatric surgery may result in a durable response by addressing obesity directly, and subsequently improve oncologic and reproductive outcomes. However, it is not known whether bariatric surgery is acceptable to this patient population.

Methods We performed a qualitative study to understand the acceptability of bariatric surgery among women of reproductive age with $\mathrm{BMI} \geq 35$ and grade $1 \mathrm{EC} / \mathrm{AH}$. Semi-structured interviews were used to explore participant perceptions towards their weight, fertility, and the possibility of bariatric surgery as part of the treatment strategy for their EC/AH.

Results Eleven participants with median age of 33 years (range 27-38) and BMI of 42.1 (35.1-56.9) were interviewed. Two (18\%) participants had grade $1 \mathrm{EC}$, and 9 (82\%) had $\mathrm{AH}$. Patients were reluctant to accept bariatric surgery as a treatment option due to 1) lack of knowledge about the procedure, 2) stigma attached to bariatric surgery, and 3) fear of the unknown. The desire to conceive was highlighted as the strongest motivator for patients to consider bariatric surgery. Their perception towards their weight, fertility and diagnosis of EC/AH were characterized by concepts of 'helplessness', 'isolation', 'frustration' and 'guilt'. We observed a significant gap in participant understanding of the complex interplay between their cancer, fertility and obesity.

Conclusions We need to provide patient-oriented counseling on implication of their weight on their cancer and fertility, before presenting bariatric surgery as a treatment option.

\section{EPV147/\#253 LIVE BIRTH, REMISSION AND RELAPSE RATES FOR FERTILITY-PRESERVING TREATMENTS OF ENDOMETRIAL ADENOCARCINOMA: A SYSTEMATIC REVIEW AND META-ANALYSIS}

S Oxley*, O Ogunbiyi, R Graham, A Olaitan. UCLH, Women's Health, London, UK

\subsection{6/ijgc-2021-IGCS.217}

Objectives Endometrial adenocarcinoma affects over 380,000 women annually, with increasing incidence primarily driven by obesity. 5-7\% of women are below 45 years at diagnosis, and many of these desire fertility-preservation rather than standard surgical treatment. This updated review aims to inform decision making in clinical practice, by evaluating the efficacies of different fertility-preserving treatments on the live birth, regression and relapse rates for women with endometrial carcinoma desiring fertility.

Methods A systematic search was performed of Medline, Embase, Central, \& Cochrane, to identify studies describing fertility-preserving treatment for endometrial cancer. Patients were divided into 3 treatment groups: systemic progestogens, intra-uterine progestogens, or hysteroscopic resection with adjuvant progestogen. A random-effects meta-analysis model was used.

Results 41 observational studies met inclusion criteria, with 1057 patients in total. The proportion of women receiving systemic progestogens who achieved a live birth was $18.1 \%$ (95\% CI 12.6-23.7\%), remission $71.5 \%$ (95\% CI $66.5-$ $76.4 \%$ ) and relapse $20.3 \%$ (95\% CI 13.1-27.4\%). For intrauterine progestogens, the proportion achieving a live birth was $13.3 \% \quad(95 \%$ CI $11.1-15.5 \%)$, remission $65.9 \% \quad(95 \%$ CI $53.0-78.8 \%$ ) and relapse $2.86 \%$ (95\% CI 0.0-9.16\%). For hysteroscopic resection, the proportion achieving a live birth was $19.1 \%$ (95\% CI $8.79-29.5 \%$ ), remission $82.7 \%$ (95\% CI 73.1-92.3\%) and relapse $6.80 \%$ (95\% CI 1.72-11.9\%).

Conclusions Although the quality of evidence is limited, these results demonstrate that hysteroscopic resection with adjuvant progestogen is associated with the highest rates of live birth and remission. This enables women considering such treatments to be fully counselled on the realistic possibilities of their desired reproductive and oncological outcomes.

\section{EPV148/\#91 OUTCOMES OF VARIOUS FERTILITY-SPARING OPTIONS FOR EARLY CERVICAL CANCER PATIENTS VERSUS ABDOMINAL RADICAL HYSTERECTOMY: ONE CANCER CENTER TEN-YEAR EXPERIENCE}

O Matylevich*, V Petukhou, I Bakinouskaya, S Taranenka. NN Alexandrov National Cancer Centre of Belarus, Gynecologic Oncology Department, Minsk, Belarus

\subsection{6/ijgc-2021-IGCS.218}

Objectives Cervical cancer (CC) is one of the most common malignant neoplasms and is diagnosed at the youngest middle 